\title{
Enteral immunonutrition versus enteral nutrition for patients undergoing esophagectomy: a randomized controlled trial
}

\author{
Xiao-Kun Li ${ }^{1 \#}$, Zhuang-Zhuang Cong ${ }^{2 \#}$, Wen-Jie $\mathrm{Wu}^{3 \#}$, Yang $\mathrm{Xu}^{4}$, Hai Zhou ${ }^{4}$, Gao-Ming $\mathrm{Wang}^{5}$, \\ Yong Qiang ${ }^{1}$, Li-Guo Luo ${ }^{1,2,3,4,5}$, Yi Shen ${ }^{1,2,3,4,5}$
}

${ }^{1}$ Department of Cardiothoracic Surgery, Jingling Hospital, Medicine School of Southeast University, Nanjing, China; ${ }^{2}$ Department of Cardiothoracic Surgery, Jingling Hospital, Medical School of Nanjing University, Nanjing, China; ${ }^{3}$ Department of Clinical Medicine, School of Medicine, Southeast University, Nanjing, China; ${ }^{4}$ Department of Cardiothoracic Surgery, Jingling Hospital, School of Clinical Medicine, Nanjing Medical University, Nanjing, China; ${ }^{5}$ Department of Thoracic Surgery, Xuzhou Central Hospital, Xuzhou School of Clinical Medicine of Nanjing Medical University, Nanjing, China

Contributions: (I) Conception and design: XK Li; (II) Administrative support: Y Shen, LG Luo; (III) Provision of study materials or patients: XK Li, Y Shen, ZZ Cong, LG Luo; (IV) Collection and assembly of data: XK Li, WJ Wu; (V) Data analysis and interpretation: XK Li, Y Xu; (VI) Manuscript writing: All authors; (VII) Final approval of manuscript: All authors.

"These authors contributed equally to this work.

Correspondence to: Yi Shen. Department of Cardiothoracic Surgery, Jingling Hospital, School of Medicine, Southeast University, Nanjing, China. Email: dryishen@nju.edu.cn; Li-Guo Luo. Department of Cardiothoracic Surgery, Jingling Hospital, Medical School of Nanjing University, Nanjing, China. Email: nanjingjingling123@163.com.

Backgroundk In recent years, immunonutrition has been introduced and proposed to have a positive
modulatory effect on inflammatory and immune responses and gut function for surgical patients, especially
for patients undergoing gastrointestinal cancer resection. We conducted this parallel-group, randomized
and double-blind clinical controlled trial to investigate the efficacy of perioperative enteral immunonutrition
(EIN) on clinical and immunological outcomes of patients undergoing esophageal resection. Methods: A randomized, parallel-group, double-blind, clinical trial was conducted between December 1, 2017 and March 1, 2018. This study enrolled 120 patients with esophageal cancer. And 112 patients were divided into two groups randomly: EIN group and enteral nutrition (EN) group. The EIN contained extra immunonutritional substrates, including a consistent combination of arginine, RNA and the omega-3 fatty acids compared with EN. Immune indicators were measured at preoperative day 7, postoperative day (POD) 1, 3, 7 and post-discharge day (PDD) 30 .

Results: There were 56 participants randomized to each group. Finally, 53 patients in EIN and 50 patients in $\mathrm{EN}$ were analyzed. Immune indicator was the primary outcome in this study. EIN yielded a significantly lower rate of CD8/CD3 (\%) at POD 3 compared with EN group (P=0.005). The rate of CD4/CD8 (\%) in EIN group was higher than that in EN group at POD3 $(\mathrm{P}=0.004)$. The serum levels of IgM at POD 3 and 7 were significantly higher in $\mathrm{EN}$ group compared with $\mathrm{EIN}$ group $(\mathrm{P}=0.025$ and $\mathrm{P}=0.009$, respectively). The rate of $\mathrm{NK}(\%)$ and the serum level of IgA were significantly higher in EIN group compared with EN group at PDD $30(\mathrm{P}=0.022$ and $\mathrm{P}=0.041$, respectively). No significant differences were found in 2-year progressionfree survival and overall survival.

Conclusions: Immunonutrition is a safe and feasible nutritional treatment, which has a positive modulatory impact on immune responses after esophagectomy. Although no significant difference was found in clinical and survival outcomes between EIN and EN groups, immunonutrition could still have a positive effect on immunological function of patients undergoing esophagectomy.

\footnotetext{
^ ORCID: 0000-0002-1432-5573.
} 


\begin{abstract}
Keywords: Enteral immunonutrition (EIN); enteral nutrition (EN); immune function; esophagectomy; randomized controlled trial
\end{abstract}

Submitted Jul 11, 2020. Accepted for publication Sep 10, 2020.

doi: 10.21037/apm-20-1399

View this article at: http://dx.doi.org/10.21037/apm-20-1399

\section{Introduction}

Esophageal cancer is the sixth most common cause of death from cancer and the eighth most common malignant tumor worldwide (1). The 5 -year overall survival rate of esophageal cancer is about $15-34 \%$, which remains poor (2). Surgery is still considered to be the preferred therapeutic option for patients with resectable esophageal cancer (3). Unsatisfactory clinical outcomes remain the problems due to the high rates of postoperative complications and morbidity, although the rapid improvements were seen in the surgical techniques (4). Meanwhile, such complications can apparently slow recovery, increase the duration of ICU and hospital stay, and ultimately rise the healthcare costs (5).

Appropriate nutrition supplement has been regarded as an important treatment to enhance the recovery of the patients who underwent esophagectomy (6). Enteral nutrition (EN) support was proved to be more effective and economical with lower rate of complications compared with paraenteral nutrition (PN), reported by several studies (79). In recent years, immunonutrition has been introduced and proposed to have a positive modulatory effect on inflammatory and immune responses and gut function for surgical patients, especially for patients undergoing gastrointestinal cancer resection $(10,11)$. Over the past years, there have been standard artificial immunonutrition products enriched in nutrients developed to stimulate the host immune function, moderate the inflammatory response and increase nitrogen balance and protein synthesis after major surgery (12). The immune-modulating substances including arginine, omega- 3 -fatty acids $(\omega-3 \mathrm{FA})$, ribose nucleic acid (RNA) were added to EN supplement to form enteral immunonutrition (EIN) (13-15). Although there have been some studies completed to investigate the impacts of EIN compared to standard EN in patients undergoing esophagectomy, it remains unclear that the EIN is feasible for improving the clinical and immunologic status compared with standard EN (15). In a published metaanalysis (16), only six articles were included and two articles contained immunological outcomes. According to the result of this meta-analysis, whether EIN could improve the clinical outcomes or biological status after esophagectomy compared to standard EN is uncertain. Therefore, it is still controversial to consider immunonutrition as a standard nutritional support in major esophageal surgery. We conducted this parallel-group, randomized and doubleblind clinical controlled trial to investigate the efficacy of preoperative and postoperative EIN versus $\mathrm{EN}$ on clinical and immunological outcomes of patients undergoing esophagectomy. We present the following article in accordance with the CONSORT reporting checklist (available at http://dx.doi.org/10.21037/apm-20-1399).

\section{Methods}

A randomized, parallel-group, double-blind, clinical trial was conducted between December 1, 2017 and March 1, 2018 at the Department of Cardiothoracic Surgery of Jingling Hospital. The study was conducted in accordance with the Declaration of Helsinki (as revised in 2013) and the Harmonized Tripartite Guideline for Good Clinical Practice from the International Conference on Harmonization. This study was reviewed and approved by the Institutional Review Board of the Jingling Hospital (approval number ChiCTR1800015951). All patients enrolled completed the informed consent form. There were 120 patients enrolled in this research after a power calculation, and 8 patients were excluded due to not progressing to surgery and not meeting the inclusion criteria. Exclusion criteria included: (I) inability to swallow; (II) a history of allergy to milk or soy; (III) acute or unstable cardiac conditions (e.g., unstable angina or symptomatic severe aortic stenosis); (IV) cardiac failure (New York Heart Association functional classes III and IV); (V) severe renal dysfunction, chronic obstructive pulmonary disease (forced expiratory volume in the first second of expiration $<60 \%$ predicted); (VI) liver dysfunction, or (VII) acute pancreatitis. The study protocol was explained to the patients with detail and informed consent were obtained from all the patients.

There were 112 patients randomly divided into two 
groups before operation (EIN group or EN group). A computer random number generation was used to do the randomization. The patient was allocated to the EIN group, if an even number was generated. Otherwise, the patient was allocated to the EN group. Care givers, participants and those assessing the outcomes were blinded to group assignment. The main surgeons (Yi Shen, XiaoKun Li, Yong Qiang), statisticians (Wen-Jie Wu, Hai Zhou), and care givers (nurses) were unaware of the group assignments. Only the nutritionist (Yang $\mathrm{Xu}$ ) who was the third part of the research known the nutritional intervention of participants. Double-blind was performed in this RCT, during which immunonutrition and standard nutrition supplement were both sponsored by the same industry (Nutricia) with same color and taste, while the EN in EIN group contained extra immunonutritional substrates, including a consistent combination of arginine, RNA (dietary nucleotides), and the omega-3 fatty acids (O3FA) compared with EN group. All the components except the extra immunonutritional substrates were exactly the same between two groups. The cost of immunonutritional substances and EN were sponsored by the Nutricia as compensation for the participants. The nutritional supplement of two groups was based on the clinical nutrition guidelines in surgery introduced by ESPEN, including preoperative, postoperative and prolonged one-month home EN (17-20). After surgery, all patients were followed up every 3 months in the first 1 year, and then every 6 months thereafter. Our follow-ups were conducted through out patient department visit. If the patient could not return to our hospital for the follow-up, we would advise the patient to perform the same assessments at local medical institutions or by phone visit. Whenever recurrence was suspected, we attempted to obtain histological or unequivocal radiological proof. Survival outcomes consisting of overall survival (OS, defined as the time between surgery and death from any cause) and disease-free survival (DFS, defined as the duration between surgery and recurrence of the disease or the death due to disease progression). The last follow-up was implemented in March 1, 2020.

\section{Nutritional protocol}

In addition to oral diet, oral nutrition or immunonutrition supplement of $500 \mathrm{~mL} /$ day via oral intake was implemented in all patients before surgery. EIN or EN (Peptisorb, Nutricia) was infused via jejunostomy tube by using an enteral feeding pump (Flocare ${ }^{\circledR}$ Infinity pump, Nutricia).
The volume of EIN and EN is $500 \mathrm{~mL} /$ bottle. A bottle $(500 \mathrm{~mL})$ of EIN and EN both contain calorie $375 \mathrm{kcal}$, protein $16 \mathrm{~g}$, carbohydrate $126 \mathrm{~g}$. The EIN contains additional immunonutritional substance including arginine $(0.13 \mathrm{~g} / 100 \mathrm{~mL}), \mathrm{RNA}$ (dietary nucleotides), and enriched omega-3 fatty acids (O3FA: 4\% alpha linolenic acid, $1.2 \%$ eicosapentaenoic acid, and $0.8 \%$ docosahexaenoic acid). The energy of $23-30 \mathrm{kcal} / \mathrm{kg}$ for postoperative days (PODs) was calculated as the patients' non-protein energy requirements by performing Henry Oxford equations (21). The protein of $1.2-1.5 \mathrm{~g} / \mathrm{kg}$ for PODs was estimated to meet the protein and nitrogen requirement. Meanwhile, adverse reactions such as diarrhea, bloating and vomiting were avoided maximumly by combining the clinical experience of clinicians.

Table 1 show the nutritional support protocol in our institution. The immunonutrition and $\mathrm{EN}$ were commenced on pre-operative day 7 by $500 \mathrm{~mL} /$ day via oral intake with energy of $750 \mathrm{kcal} /$ day in addition to diet before surgery. Jejunal enteral feeding was commenced on POD 1 at $20-25 \mathrm{~mL} /$ hour. Oral feeding resumed following bedside assessment of swallow for patients who underwent esophagectomy with cervical anastomosis at PODs 5, meanwhile liquid diet was implemented by oral intake with 100-200 g. From that time, patients recommenced a graded introduction to diet from sips to free fluids, light diet (soup/jelly/ice-cream) by day 6. Patients of HEN were instructed to independently administer jejunostomy feeding at home if discharge and jejunostomy tubes were removed at post-discharge day (PDD) 30 uniformly. The patients were regarded as lost for follow-up, if their jejunostomy tubes were removed after surgery and before PDD30 due to complications or other reasons.

The discharge criteria were listed as follows: patients can move autonomously and be able to consume soft diet by oral intake; no fever and other complications; adequate pain control with oral analgesics; chest tube(s) and other surgical drainages were removed. On 30 days' follow-up after discharge immune status of patients were measured.

\section{Surgical methods}

All the patients who received esophagectomy, which comprised open surgery, video-assisted thoracic surgery (VATS), and robot-assisted thoracic surgery (RATS), underwent McKeown esophagectomy with cervical anastomosis. During VATS and RATS, the mediastinum was dissected from the diaphragm to the apex of the chest 
Table 1 Nutritional support pathway

\begin{tabular}{|c|c|}
\hline Days & Enteral immunonutrition in EIN group or enteral nutrition in EN group \\
\hline 1 & Amino acids of $1.5 \mathrm{~g} / \mathrm{kg}$ and energy of $23-30 \mathrm{kcal} / \mathrm{kg}$ provided by $\mathrm{PN}$ \\
\hline \multirow{2}{*}{2} & $500-750 \mathrm{~mL}$ ONS by $25-35 \mathrm{~mL} /$ hour via jejunostomy tube \\
\hline & Amino acids of $1.0 \mathrm{~g} / \mathrm{kg}$ and energy of $20 \mathrm{kcal} / \mathrm{kg}$ provided by $\mathrm{PN}$ \\
\hline 3 & Amino acids of $1.0 \mathrm{~g} / \mathrm{kg}$ and energy of $15 \mathrm{kcal} / \mathrm{kg}$ provided by PN \\
\hline \multirow{2}{*}{4} & $800-1,200 \mathrm{~mL}$ ONS by $45-60 \mathrm{~mL} /$ hour via jejunostomy tube \\
\hline & Amino acids of $0.8 \mathrm{~g} / \mathrm{kg}$ and energy of $10 \mathrm{kcal} / \mathrm{kg}$ provided by PN \\
\hline 5 & Water soluble $100-150 \mathrm{~g}$ by oral intake \\
\hline 6 & $1,500-1,800 \mathrm{~mL}$ ONS by $60-80 \mathrm{~mL} /$ hour via jejunostomy tube \\
\hline \multirow{3}{*}{$7 \sim$} & Light diet (soup/ jelly/ ice-cream) $200-300 \mathrm{~g}$ by oral intake \\
\hline & $1,500-2,000 \mathrm{~mL}$ ONS by $70-90 \mathrm{~mL} /$ hour via jejunostomy tube \\
\hline & The patients were instructed to administer jejunostomy feeds independently at home (after discharge) \\
\hline $\begin{array}{l}\text { From discharge to } 1 \\
\text { month }\end{array}$ & $\begin{array}{l}\text { Light diet was introduced at the first week and normal diet was introduced after first week, with ONS of } 500 \mathrm{~mL} / \mathrm{day} \\
\text { via jejunostomy tube or oral intake }\end{array}$ \\
\hline 30 & Measurement of immune indicators \\
\hline
\end{tabular}

EIN, enteral immunonutrition; EN, enteral nutrition; ONS, oral nutrition supplement; PN, parenteral nutrition.

with gentle separation of fat, nodes, the azygos vein, and the esophagus. The thorax was then closed with the patient placed in a supine position. The abdomen was explored through a midline incision, and the right gastric and right gastroepiploic arteries were preserved to provide vascular supply to the gastric tube, and fat and nodes along the celiac trunk and the upper part of the abdominal aorta were removed. After the gastric tube was created, the jejunostomy tubes were inserted through the left upper abdomen into the jejunum. To expose the operative field, the patient was laid in a supine position with the neck extended at the very beginning and the head turned to the right afterward. An oblique incision was made through the skin and muscles on the left side of the neck, and the prepared gastric tube was gently pulled up with the specimen through the posterior mediastinum and removed to the neck. Finally, the esophagus was resected and the anastomosis was sutured. The open operation started with a left thoracotomy, after which the esophagus was divided gently from the diaphragm to the apex of the chest. The stomach was explored through an incision in the left diaphragm and a gastric tube was created, followed by the same VATS and the RATS procedures.

\section{Clinical variables}

The patient characteristics, including sex, age, BMI, preoperative complications, preoperative thoracic complications, receipt of neoadjuvant chemotherapy, operation time, type of operation, operative blood loss, pathological stage according to Union for International Cancer Control (UICC) and postoperative hospital stay, were recorded. The postoperative infectious complications such as pneumonia, surgical site infection and anastomotic leakage and postoperative complications classified according to Clavien-Dindo were also collected.

\section{Immune indicators}

Immune parameters, which were the primary outcomes, 
were measured at preoperative day 1, 5, 7 and at PDD 30. The proportion and number of T-cells, T-cell subsets and NK (natural killer) cells were recorded. Immunoglobulin $\mathrm{G}, \mathrm{M}$ and $\mathrm{A}$ ( $\operatorname{IgG}, \operatorname{Ig} M$ and $\operatorname{IgA}$ ) were also included. Meanwhile, the level of interleukin-6 (IL-6) and C-reactive protein (CRP) were measured. The immunological markers were selected according to the previous studies associated with immunonutrition in patients undergoing esophagectomy (12). The proportion of lymphocyte can reflect the cellular immunity and the immunoglobulins can reveal the status of humoral immunity. The inflammatory biomarkers including CRP and IL-6 were also chosen as immunological markers in this study.

\section{Statistical analysis}

A power calculation was performed prior to start of the trial. A preliminary test including 60 patients in our institution was completed to make an estimation. On the basis of this estimation, the level of IgG in EIN group increased $1.52 \pm 1.95 \mathrm{~g} / \mathrm{L}$ (mean \pm standard deviation) at PDD30 and the level of $\operatorname{IgG}$ in the patients of EN group increased $0.42 \mathrm{~g} / \mathrm{L}$ (mean) at PDD30. A sample size of 50 participants per group for 0.8 power was required to detect the assumed difference between means with a 0.05 significant level. $20 \%$ discontinue treatment and/or loss to follow-up was assumed for each group. Finally, a sample size of 60 subjects was determined in each group. R 3.6.1 software was implemented to calculate the sample size. The continuous data are presented as the mean \pm standard deviation (SD). The differences of continuous variables between two groups were assessed using Student's $t$-test or repeated measures two-way ANOVA. Pearson's $\chi^{2}$ or Fisher's exact tests were conducted to compare the categorical variables between two groups. Overall survival (OS) and progression-free survival (PFS) were determined by Kaplan-Meier actuarial analysis and log-rank tests were implemented to analyze the differences of survival curves between two groups. $\mathrm{P}<0.05$ was considered statistically significant. Statistical program SPSS 21.0 software (SPSS, Chicago, IL) was employed to analyze the data.

\section{Results}

There were 120 patients assessed for eligibility. Three patients not meeting inclusion criteria, 2 patients declining to participate, 2 patients being unable to attend clinic and 1 missing in preadmission clinic were excluded. There were
56 patients randomized into each group, respectively. One randomized patient in EIN group and 2 in EN group were withdrawn because of their incorrect intervention. There were 55 patients receiving allocated intervention in EIN and 54 in EN. Until PDD30 after surgery, skin inflammation occurred in 2 patients in EIN group and 2 in EN group occurred. Their jejunostomy tubes were then removed, which was considered as discontinued intervention. Another 2 patients in EN group were lost for follow-up due to one unable to be contacted and another withdrawing consent. Finally, 53 patients in EIN and 50 patients in EN were retained for final analysis at PDD30 (Figure 1).

Table 2 displayed the baseline characteristics of the participants enrolled in this study. No significant differences were seen in sex, age, BMI, incidence of hypertension, diabetes, and preoperative thoracic complications, pathological stage or receipt of neoadjuvant chemotherapy between the EIN group and EN group.

Table 3 demonstrated the operative data and short-term clinical outcomes. The differences in operation time, type of operation, or operative blood loss between two groups were not significant. As to short-term clinical outcomes, no significant differences were found in the incidence of postoperative complications (12 in EIN group and 11 in EN group) or postoperative hospital stay between the two groups. EIN group yielded a lower rate of infectious complications compared with EN group (EIN: $15 \%$ and $\mathrm{EN}$ : $22 \%, \mathrm{P}=0.45$ ) but no significant difference was found between EIN group and EN group. Meanwhile, there were no significant differences in the incidence of pneumonia, surgical site infection or anastomotic leakage $(\mathrm{P}=0.76$, $\mathrm{P}=0.52$ and $\mathrm{P}=0.71$, respectively). The differences between two groups in adverse events, such as bloating, diarrhea and vomiting, were also not significant.

Immune parameters were shown in Table 4. The serum indicators were all collected at PRD 7, POD 1, 3, 7 and PDD 30. Comparing the baseline serum level between two groups, no significant differences were found in immune indicators at PRD 7 between two groups. EIN group yielded a significantly lower rate of $\mathrm{CD} 8 / \mathrm{CD} 3$ (\%) at POD 3 compared with $\mathrm{EN}$ group $(\mathrm{P}=0.005)$. Overall, the rate of CD8/CD3 (\%) in the EIN group tended to be higher than that in the EN group $(\mathrm{P}=0.069$; Figure 2). The EIN group yielded a higher rate of CD4/CD8 (\%) compared with EN group at POD3 $(\mathrm{P}=0.004)$. The serum levels of IgM at POD 3 and 7 in EN group were significantly higher than those in EIN group ( $\mathrm{P}=0.025$ and $\mathrm{P}=0.009$, respectively). Overall, EIN group yielded a lower IgM level compared 


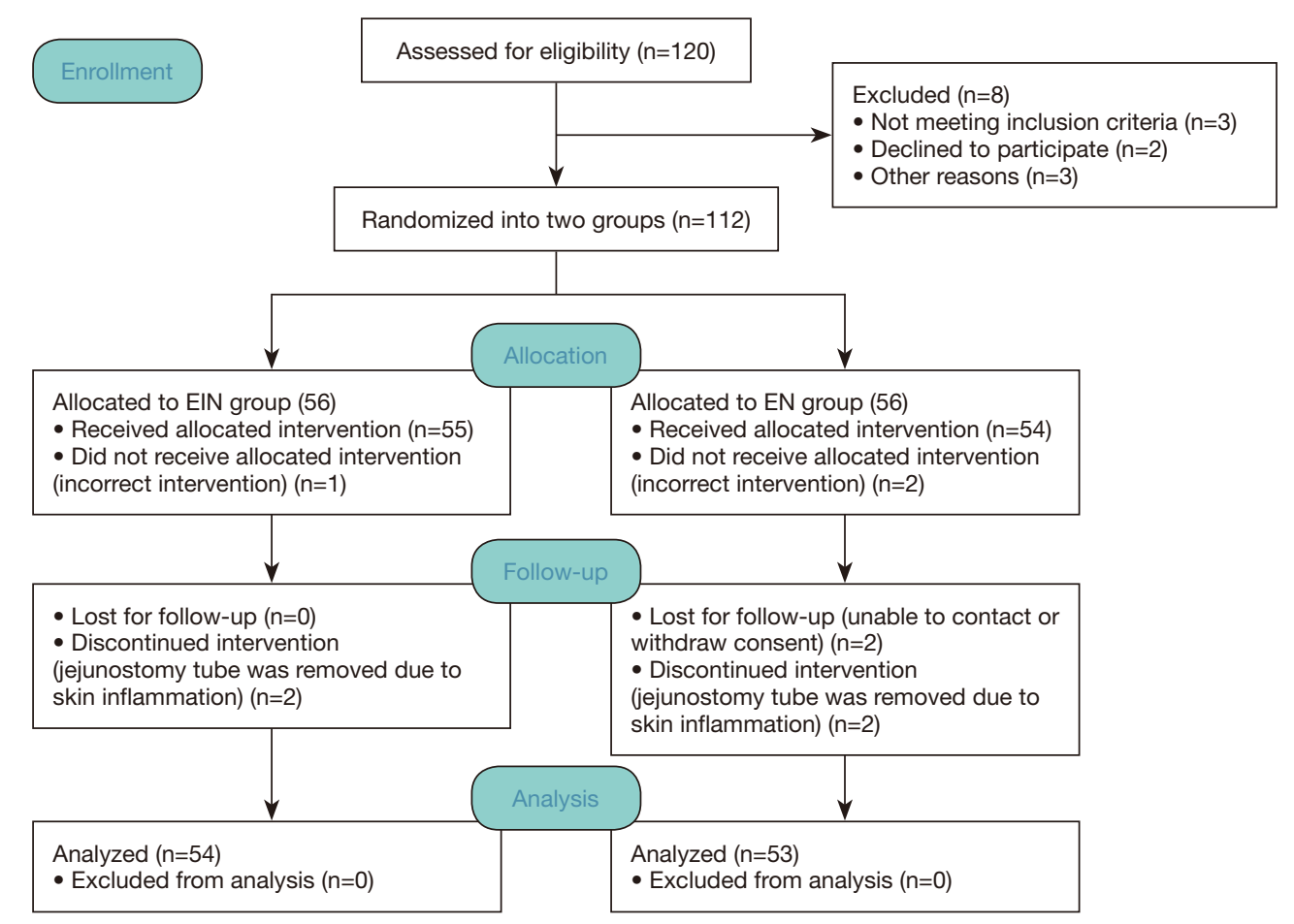

Figure 1 Flowchart. EIN, enteral immunonutrition; EN, enteral nutrition.

with EN group ( $\mathrm{P}=0.034$; Figure 3). The rate of NK (\%) and the serum level of IgA were significantly higher in EIN group compared with EN group at PDD $30(\mathrm{P}=0.022$ and $\mathrm{P}=0.041$, respectively).

There was no significant difference in 2-year OS comparing two groups (2-year overall survival rate: $74.7 \%$ and 69.2\%, respectively; Log-rank $\mathrm{P}=0.45$ ) (Figure 4). Meanwhile, no significant difference was found in 2-year PFS (2-year progression-free survival rate: $67.0 \%$ and $63.0 \%$, respectively; Log-rank $\mathrm{P}=0.59$ ) (Figure 5).

\section{Discussion}

Esophageal cancer, characterized as a common malignant tumor, has been associated with impairment of low immune function (22). Meanwhile, the surgical trauma would cause further decrease in immune function after operation, making the residual tumor cells easily escape from immune surveillance. This could finally result in the early relapse of cancer (23). Some randomized control trials suggested that patients may benefit from EIN before or after resectional surgery (10-12). Furthermore, ESPEN guideline strongly recommend the use of immune-nutrition for major cancer surgery, which was based mostly on randomized controlled studies on total gastrectomy for gastric carcinoma (17). Therefore, the patients undergoing esophagectomy may also benefit from the addition of immune-enhancing compounds in enteral feeding due to their impaired immune response. Arginine, $\omega-3 \mathrm{FA}$ and RNA are the most common immunemodulating substances added to enteral feeds. Arginine is characterized as a precursor to nitric oxide synthesis, which becomes essential amino acid during recovery and growth after injury (11). Omega-3 fatty acids have the ability to modulate the synthesis of various eicosanoids by exerting immunomodulatory, vasodilatatory, and anti-inflammatory properties (12). RNA nucleotides are considered to play an important role in the immune response since RNA nucleotide-deficient diets have resulted in the diminished T-cell responses and decreased production of interleukin-2 (10). However, the clinical efficacy of EIN for patients undergoing esophagectomy was still controversial, although the immune-related compounds might have theoretical benefits. Since only 6 randomized controlled trials were conducted to determine the efficiency of immunonutrition for patients undergoing esophagectomy. And only 5 out of the 6 studies investigated the serum level of immune indicators perioperatively. However, no studies reported the rate of nature-killer cell and only one study 
Table 2 Clinical characteristics

\begin{tabular}{|c|c|c|c|}
\hline Variables & EIN & EN & $P$ value \\
\hline Cases & 53 & 50 & \\
\hline Age (year) & $62.13 \pm 6.51$ & $61.52 \pm 5.97$ & 0.62 \\
\hline Sex & & & 0.82 \\
\hline Male & 36 & 35 & \\
\hline Female & 17 & 15 & \\
\hline BMI & $23.55 \pm 3.26$ & $23.65 \pm 4.10$ & 0.89 \\
\hline Diabetes & & & 0.57 \\
\hline Yes & 6 & 4 & \\
\hline No & 47 & 46 & \\
\hline Hypertensior & & & 0.55 \\
\hline Yes & 5 & 7 & \\
\hline No & 48 & 43 & \\
\hline \multicolumn{3}{|c|}{ Preoperative thoracic complications } & 0.61 \\
\hline Yes & 1 & 2 & \\
\hline No & 52 & 48 & \\
\hline \multicolumn{3}{|c|}{ Receipt of neoadjuvant chemotherapy } & 0.58 \\
\hline YES & 9 & 6 & \\
\hline No & 44 & 44 & \\
\hline \multicolumn{3}{|c|}{ Pathological stage (UICC) } & 0.9 \\
\hline I & 14 & 11 & \\
\hline II & 23 & 22 & \\
\hline III & 12 & 14 & \\
\hline IV & 4 & 3 & \\
\hline
\end{tabular}

BMI, body mass index; UICC, Union for International Cancer Control; EIN, enteral immunonutrition; EN, enteral nutrition.

investigated the serum level of immunoglobulin.

The study of Satoshi Aiko et al. (12) demonstrated that EN group yielded significantly higher IL-8 level on POD5. EIN group yielded a significantly larger proportion of T-cells on POD 1 and 7 and smaller proportion of B-cells on POD 1 compared with EN group. However, there was no significant difference in the NK-cell (\%) between EN group and EIN group. Ryan et al. (14) reported that IL-8 levels were significantly $(\mathrm{P}<0.05)$ lower on POD 7 and 14 in the EIN group. Kitagawa et al. (11) reported that the TNF- $\alpha$ levels in the EN group were significantly higher than those in the EIN group $(\mathrm{P}=0.033)$. The study of Sakurai et al. (13) showed that the total peripheral
Table 3 Surgical data and postoperative clinical outcomes

\begin{tabular}{|c|c|c|c|}
\hline Characteristics & EIN & EN & $P$ value \\
\hline Cases & 53 & 50 & \\
\hline Type of operation & & & 0.78 \\
\hline RATS & 12 & 9 & \\
\hline VATS & 37 & 38 & \\
\hline Open operation & 4 & 3 & \\
\hline Operation time & $214.98 \pm 54.63$ & $209.88 \pm 51.33$ & 0.63 \\
\hline Operative blood loss (mL) & $108.23 \pm 61.75$ & $113.64 \pm 65.14$ & 0.67 \\
\hline Postoperative complicatio & & & 0.56 \\
\hline Minor (CDC Grade II) & 7 & 6 & \\
\hline Major (CDC Grade III) & 5 & 4 & \\
\hline Major (CDC Grade IV) & 0 & 1 & \\
\hline Infectious complications & 8 & 11 & 0.45 \\
\hline Pneumonia & 5 & 6 & 0.76 \\
\hline Surgical site infection & 4 & 6 & 0.52 \\
\hline Anastomotic leakage & 3 & 4 & 0.71 \\
\hline Adverse events & 20 & 18 & 0.55 \\
\hline Bloating & 13 & 15 & 0.66 \\
\hline Diarrhea & 6 & 4 & 0.74 \\
\hline Vomiting & 7 & 4 & 0.53 \\
\hline $\begin{array}{l}\text { Postoperative hospital } \\
\text { stay }\end{array}$ & $13.34 \pm 5.94$ & $14.37 \pm 7.28$ & 0.43 \\
\hline
\end{tabular}

RATS, robot-assisted thoracic surgery; VATS, video-assisted thoracic surgery; EIN, enteral immunonutrition; EN, enteral nutrition.

lymphocyte counts, the percent lymphocyte fraction, and the percentage of B-cell fraction in the EIN group at $3 \leq$ day $\leq 7$ after operation was significantly higher than those in the EN group, while the EIN group yielded a significantly lower percentage of T-cell fraction at 3 day after operation compared with EN group. For immunoglobulin, the serum level of $\mathrm{IgG}$ in the EIN group at POD3 was significantly higher than that in EN group. Only the study of Kanekiyo et al. (10) compared the FPS and OS between two groups, and no significant differences were found in overall survival and progression-free survival outcomes $(\mathrm{P}=0.19$ and $\mathrm{P}=0.13$, respectively).

Our findings indicate that the infectious complications were less common in the EIN group. However, these differences did not reach statistical significance, which 
Table 4 Immune indicators

\begin{tabular}{|c|c|c|c|c|c|c|}
\hline & PRD7 & POD1 & POD3 & POD7 & POD30 & $P$ value \\
\hline EIN & $64.26 \pm 9.22$ & $49.84 \pm 10.12$ & $55.24 \pm 9.29$ & $60.11 \pm 6.75$ & $65.35 \pm 7.37$ & \\
\hline EN & $65.85 \pm 8.76$ & $48.23 \pm 11.85$ & $58.42 \pm 14.47$ & $62.81 \pm 9.57$ & $65.99 \pm 6.48$ & \\
\hline CD4 (\%) & & & & & & 0.58 \\
\hline EN & $38.88 \pm 8.97$ & $29.87 \pm 5.86$ & $32.31 \pm 9.04$ & $38.56 \pm 8.29$ & $38.63 \pm 6.85$ & \\
\hline CD8 (\%) & & & & & & 0.069 \\
\hline EIN & $23.12 \pm 7.45$ & $19.75 \pm 8.12$ & $17.96 \pm 7.55^{\star \star}$ & $20.33 \pm 5.95$ & $26.00 \pm 7.66$ & \\
\hline EN & $23.01 \pm 9.21$ & $21.22 \pm 11.85$ & $23.81 \pm 12.52^{\star \star}$ & $22.54 \pm 9.30$ & $26.21 \pm 11.47$ & \\
\hline EN & $2.06 \pm 1.43$ & $1.81 \pm 3.94$ & $1.73 \pm 0.82^{\star \star}$ & $2.11 \pm 1.23$ & $1.95 \pm 1.33$ & \\
\hline NK (\%) & & & & & & 0.54 \\
\hline EIN & $17.75 \pm 9.46$ & $21.65 \pm 9.75$ & $17.50 \pm 6.33$ & $15.85 \pm 7.93$ & $19.40 \pm 7.02^{*}$ & \\
\hline EN & $18.10 \pm 7.02$ & $20.19 \pm 10.38$ & $15.00 \pm 9.09$ & $14.13 \pm 9.21$ & $15.83 \pm 8.49^{\star}$ & \\
\hline $\lg G(g / L)$ & & & & & & 0.25 \\
\hline EIN & $12.38 \pm 2.83$ & $9.91 \pm 2.14$ & $9.07 \pm 2.04$ & $9.44 \pm 3.26$ & $13.22 \pm 3.77$ & \\
\hline EN & $11.68 \pm 1.68$ & $9.71 \pm 1.81$ & $8.71 \pm 1.81$ & $8.90 \pm 1.55$ & $12.62 \pm 2.75$ & \\
\hline $\lg M(g / L)$ & & & & & & 0.034 \\
\hline EN & $2.15 \pm 0.41$ & $1.94 \pm 0.64$ & $1.58 \pm 0.52$ & $2.00 \pm 0.69$ & $2.11 \pm 0.69^{*}$ & \\
\hline CRP (mg/L) & & & & & & 0.98 \\
\hline EIN & $2.83 \pm 4.02$ & $54.89 \pm 33.09$ & $122.15 \pm 65.25$ & $49.52 \pm 31.55$ & $4.50 \pm 8.09$ & \\
\hline EN & $2.60 \pm 3.97$ & $61.26 \pm 26.44$ & $115.32 \pm 59.14$ & $54.33 \pm 40.14$ & $7.26 \pm 11.92$ & \\
\hline IL-6 (pg/mL) & & & & & & 0.42 \\
\hline EIN & $6.29 \pm 3.57$ & $210.37 \pm 120.21$ & $56.47 \pm 47.97$ & $25.85 \pm 14.23$ & $3.88 \pm 2.18$ & \\
\hline EN & $6.53 \pm 4.15$ & $201.25 \pm 147.20$ & $62.34 \pm 45.92$ & $23.22 \pm 16.45$ & $4.49 \pm 3.52$ & \\
\hline
\end{tabular}

${ }^{*}, \mathrm{P}<0.05 ;{ }^{* *}, \mathrm{P}<0.01$ (Student's $t$-test). $\mathrm{P}$ value (repeated measures two-way ANOVA). PRD, preoperative day; POD, postoperative day; PDD, Post-discharge day; IgG, immunoglobulin G; IgM, immunoglobulin M; IgA, immunoglobulin A; NK, natural killer; IL-6, interleukin-6; CRP, C-reactive protein.

may be due to the small number of subjects enrolled in this study. In our study, the rate of CD8 in EIN group is significantly lower than that in EN group, which result in a decreasing rate of CD4/CD8 in EIN group at POD3. Meanwhile, the rate of CD4 in EIN group tended to be higher than that in $\mathrm{EN}$ group $(\mathrm{P}=0.12)$ at $\mathrm{POD}$, though 


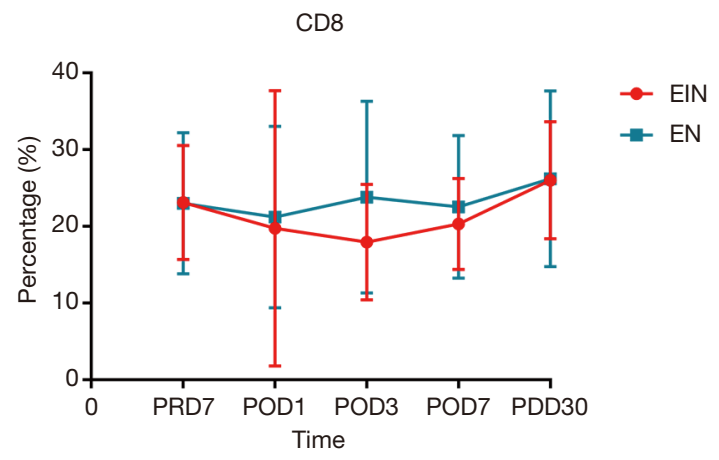

Figure 2 Continuous values are presented as means \pm SD. Overall, percentages of CD8 (\%) in the EIN group were tended to be lower than those in the EN group $(\mathrm{P}=0.069)$; EIN, enteral immunonutrition; EN, enteral nutrition.

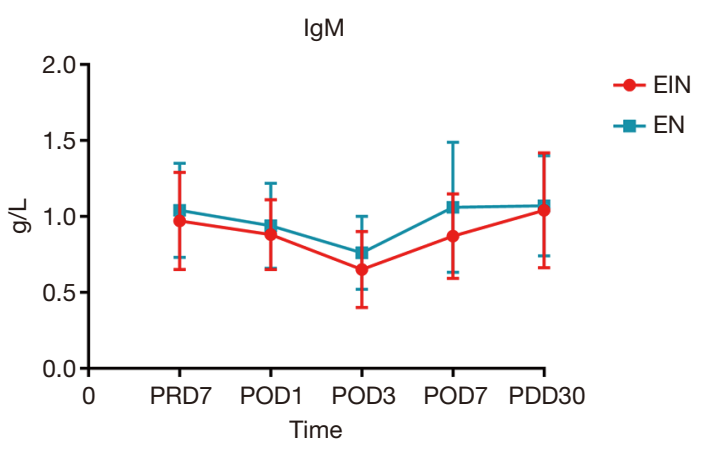

Figure 3 Continuous values are presented as means \pm SD. Overall, serum levels of $\operatorname{IgM}$ in the EIN group were tended to be lower than those in the EN group $(\mathrm{P}=0.034)$; EIN, enteral immunonutrition; EN, enteral nutrition; IgM, immunoglobulin $M$.

there was no significant difference. The results imply that EIN group may yield a better humoral immunity, while EN group might yield a more active cellular immunity. On the same day, the rate of NK cell in EIN group tended to be higher than that in EN group $(\mathrm{P}=0.11)$, which indicate that EIN may yield a better nonspecific cellular immunity. The levels of IgM in EN group were significant higher than that in EIN group at POD 3 and 7. IgM is characterized as the earliest immunoglobulin increasing in the recent infection, which reflects inflammatory responses in humanity. The increasing level of $\operatorname{IgM}$ in EN group could due to the higher incidence of infectious complications in EN group compared with EIN group, which indicate that EIN may have a positive modulatory effect on inflammatory responses and improve short-term outcomes (10). The EIN group

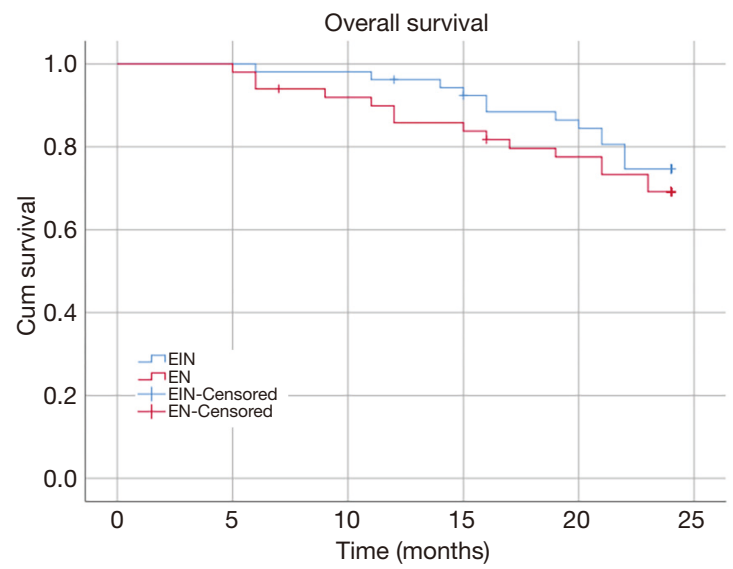

Figure 4 Overall survival. Two-year overall survival rate: $74.7 \%$ and $69.2 \%$, respectively; Log-rank P=0.45; EIN, enteral immunonutrition; EN, enteral nutrition.

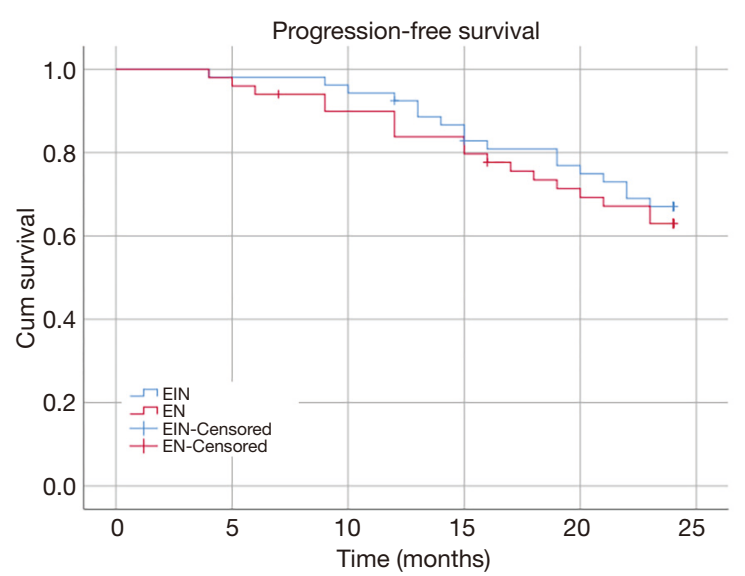

Figure 5 Progression-free survival. Two-year progression-free survival rate: $67.0 \%$ and $63.0 \%$, respectively; Log-rank $\mathrm{P}=0.59$; EIN, enteral immunonutrition; EN, enteral nutrition.

yielded a significantly higher rate of NK cell and the level of IgA at POD30 compared with $\mathrm{EN}$, which demonstrate that EIN may yield a better nonspecific cellular immunity and gut function at POD30 since IgA is the main antibody in mucosal infection (24). According to the results of repeated measures two-way ANOVA, only the level of IgM in EIN group overall tended to be lower than that in EN group ( $\mathrm{P}=0.034)$. Overall, the rate of CD8 in EIN group tended to be less than that in EN group. In conclusion, EIN yields a better humoral immunity before POD7, while EN yields a better specific cellular immunity. However, a more active nonspecific cellular immunity can be found 
in EIN group. At POD30, EIN may yield a better gut function due to the increasing level of $\operatorname{IgA}(24,25)$. The cost of immunonutritional substances and EN were sponsored by the Nutricia as compensation for the participants. Therefore, the cost-effect was not explored in this study.

\section{Conclusions}

Immunonutrition is a safe and feasible nutritional treatment, which has a positive modulatory impact on immune responses after esophagectomy. Although no significant difference was found in clinical and survival outcomes between EIN and EN groups, immunonutrition could still have a positive effect on immunological function of patients undergoing esophagectomy.

\section{Limitation}

There were no significant differences in progression-free or overall survival between EIN and EN group, which may be due to the small number of subjects enrolled in the study and the short length of survival follow-up. Thus, a longer follow-up and larger number of cohorts were needed to investigate the feasibility and effect of immunonutrition in patients undergoing esophageal cancer resection.

\section{Acknowledgments}

Funding: This work was supported by the National Natural Science Foundation of China (No. 81172032), the Natural Science Foundation of Jiangsu Province (BK20181239), the Science and technology program of Xu Zhou (No. KCI8037) and the Xuzhou Clinical Technology Key Research Project (No. 2019GG021). We thank all the members of department of cardiothoracic surgery in our hospital that participated in this research. We also thank Xiao-Kun Li and Wen-Jie Wu for their advice on statistical analysis.

\section{Footnote}

Reporting Checklist: The authors have completed the CONSORT reporting checklist. Available at http://dx.doi. org/10.21037/apm-20-1399

Data Sharing Statement: Available at http://dx.doi. org/10.21037/apm-20-1399

Conflicts of Interest: All authors have completed the ICMJE uniform disclosure form (available at http://dx.doi. org/10.21037/apm-20-1399). The authors have no conflicts of interest to declare.

Ethical Statement: The authors are accountable for all aspects of the work in ensuring that questions related to the accuracy or integrity of any part of the work are appropriately investigated and resolved. The study was conducted in accordance with the Declaration of Helsinki (as revised in 2013) and the Harmonized Tripartite Guideline for Good Clinical Practice from the International Conference on Harmonization. This study was reviewed and approved by the Institutional Review Board of the Jingling Hospital (approval number ChiCTR1800015951). All patients enrolled completed the informed consent form. The study outcomes will not affect the future patient management. This study is based on data retrieved from a hospital medical record system. All personal data have been protected and secured according to current national and international laws.

Open Access Statement: This is an Open Access article distributed in accordance with the Creative Commons Attribution-NonCommercial-NoDerivs 4.0 International License (CC BY-NC-ND 4.0), which permits the noncommercial replication and distribution of the article with the strict proviso that no changes or edits are made and the original work is properly cited (including links to both the formal publication through the relevant DOI and the license). See: https://creativecommons.org/licenses/by-nc-nd/4.0/.

\section{References}

1. Pennathur A, Gibson MK, Jobe BA, et al. Oesophageal carcinoma. Lancet 2013;381:400-12.

2. Deng HY, Wang WP, Wang YC, et al. Neoadjuvant chemoradiotherapy or chemotherapy? A comprehensive systematic review and meta-analysis of the options for neoadjuvant therapy for treating oesophageal cancer. Eur J Cardiothorac Surg 2017;51:421-31.

3. Wang H, Shen Y, Feng M, et al. Outcomes, quality of life, and survival after esophagectomy for squamous cell carcinoma: A propensity score-matched comparison of operative approaches. J Thorac Cardiovasc Surg 2015;149:1006-5.e4.

4. Berkelmans GHK, Fransen L, Weijs TJ, et al. The longterm effects of early oral feeding following minimal invasive esophagectomy. Dis Esophagus 2018;31:1-8. 
5. Steenhagen E, van Vulpen JK, van Hillegersberg R, et al. Nutrition in peri-operative esophageal cancer management. Expert Rev Gastroenterol Hepatol 2017;11:663-72.

6. Zheng R, Devin CL, Pucci MJ, et al. Optimal timing and route of nutritional support after esophagectomy: A review of the literature. World J Gastroenterol 2019;25:4427-36.

7. Klevebro F, Johar A, Lagergren J, et al. Outcomes of nutritional jejunostomy in the curative treatment of esophageal cancer. Dis Esophagus 2019;32:doy113.

8. Elshaer M, Gravante G, White J, et al. Routes of early enteral nutrition following oesophagectomy. Ann R Coll Surg Engl 2016;98:461-7.

9. Li XK, Cong ZZ, Wu WJ, et al. Efficacy of 4 wk of home enteral feeding supplementation after esophagectomy on immune function: A randomized controlled trial. Nutrition 2020;77:110787.

10. Kanekiyo S, Takeda S, Iida M, et al. Efficacy of perioperative immunonutrition in esophageal cancer patients undergoing esophagectomy. Nutrition 2019;59:96-102.

11. Kitagawa H, Namikawa T, Yatabe T, et al. Effects of a preoperative immune-modulating diet in patients with esophageal cancer: a prospective parallel group randomized study. Langenbecks Arch Surg 2017;402:531-8.

12. Aiko S, Yoshizumi Y, Ishizuka T, et al. Enteral immunoenhanced diets with arginine are safe and beneficial for patients early after esophageal cancer surgery. Dis Esophagus 2008;21:619-27.

13. Sakurai Y, Masui T, Yoshida I, et al. Randomized clinical trial of the effects of perioperative use of immune-enhancing enteral formula on metabolic and immunological status in patients undergoing esophagectomy. World J Surg 2007;31:2150-7; discussion 8-9.

14. Ryan AM, Reynolds JV, Healy L, et al. Enteral nutrition enriched with eicosapentaenoic acid (EPA) preserves lean body mass following esophageal cancer surgery: results of a double-blinded randomized controlled trial. Ann Surg 2009;249:355-63.

15. Mudge LA, Watson DI, Smithers BM, et al. Multicentre factorial randomized clinical trial of perioperative immunonutrition versus standard nutrition for patients undergoing surgical resection of oesophageal cancer. $\mathrm{Br} \mathrm{J}$ Surg 2018;105:1262-72.

16. Li XK, Zhou H, Xu Y, et al. Enteral immunonutrition versus enteral nutrition for patients undergoing oesophagectomy: a systematic review and meta-analysis. Interact Cardiovasc Thorac Surg 2020;30:854-62.

17. Weimann A, Braga M, Carli F, et al. ESPEN guideline: Clinical nutrition in surgery. Clin Nutr 2017;36:623-50.

18. Arends J, Bachmann P, Baracos V, et al. ESPEN guidelines on nutrition in cancer patients. Clin Nutr 2017;36:11-48.

19. Li C, Ferri LE, Mulder DS, et al. An enhanced recovery pathway decreases duration of stay after esophagectomy. Surgery 2012;152:606-14; discussion 14-6.

20. Lee L, Li C, Robert N, et al. Economic impact of an enhanced recovery pathway for oesophagectomy. Br J Surg 2013;100:1326-34.

21. Ramirez-Zea M. Validation of three predictive equations for basal metabolic rate in adults. Public Health Nutr 2005;8:1213-28.

22. Helminen O, Mrena J, Sihvo E. Securing enteral nutrition with routine feeding jejunostomy after esophagectomy: lost effort or a life saver? J Thorac Dis 2019;11:636-7.

23. Ling Y, Chen J, Tao M, et al. A pilot study of nimotuzumab combined with cisplatin and 5-FU in patients with advanced esophageal squamous cell carcinoma. J Thorac Dis 2012;4:58-62.

24. Schantz SP, Liu FJ, Taylor D, et al. The relationship of circulating IgA to cellular immunity in head and neck cancer patients. Laryngoscope 1988;98:671-8.

25. Shalapour S, Lin XJ, Bastian IN, et al. Inflammationinduced IgA+ cells dismantle anti-liver cancer immunity. Nature 2017;551:340-5.
Cite this article as: $\mathrm{Li} \mathrm{XK}$, Cong $\mathrm{ZZ}, \mathrm{Wu} \mathrm{WJ}, \mathrm{Xu} \mathrm{Y}$, Zhou H, Wang GM, Qiang Y, Luo LG, Shen Y. Enteral immunonutrition versus enteral nutrition for patients undergoing esophagectomy: a randomized controlled trial. Ann Palliat Med 2021;10(2):1351-1361. doi: 10.21037/apm-20-1399 